\title{
Research on textile materials for X-ray shielding
}

\author{
Dong Liang ${ }^{1 \text { st, } 1}$, Fu Shen ${ }^{1 \text { st, } 1}$, Zizhen Bao ${ }^{1}$, Yuchen Liu ${ }^{1}$, Honghui Li*1,a \\ ${ }^{1}$ China Institute for Radiation Protection, P.O. Box 120, Taiyuan 030006 , Shanxi, P.R. China
}

\begin{abstract}
X-ray radioactive rays are widely used with the continuous development of radioactive medicine and nuclear technology applications, as well as lead shielding material pollutions new no lead shielding material was needed. In this paper, the main properties of metal tungsten and bismuth as X-ray shielding materials were studied for the protection people avoid the $150 \mathrm{kV}$ X-rays by the Monte Carlo method is used to study. According to simulation with $2 \mathrm{~kg} / \mathrm{m}^{2}$, results show that performance of single metal material tungsten iron is superior to that of bismuth material. Tungsten-bismuth better than bismuth-tungsten with the case of equal-quality double-layer metal. The protection performance is better when the metal-mixed tungsten-bismuth ratio is 0.5 : 0.5 or the tungsten ratio is large. The research provides effective support for the development of textile radiation protection materials.
\end{abstract}

\section{Introduction}

In recent years, with the continuous development of national defense scientific research, radioactive medicine, and nuclear technology applications, various radioactive rays have been widely used. X-ray is a type of ionizing radiation, has been used in material $\mathrm{X}$-ray inspection, human X-ray fluoroscopy, and X-ray analysis. Achieve successful application. While using radiation to make profits, the damage to the human body and the destruction of the environment by the rays are gradually recognized by people. Long-term exposure of staff to Xrays can cause damage to the gonads, breast, hematopoietic bone marrow, etc. Excessive doses can even cause cancer, posing a serious threat to the human body. Therefore, the research on anti-X-ray materials has become an important subject. However, traditional X-ray shielding materials are mainly lead-containing vulcanized rubber and metal film materials. It has the disadvantages of high lead toxicity, environmental pollution, weak X-ray absorption area, strong scattering of low-energy X-rays, and bulky materials. Rubber materials have poor air permeability, require vulcanization processing, are not easy to prepare thin and light products, vulcanized rubber cannot be recycled, and other shortcomings. Lead-free nuclear protection materials have become an inevitable trend.

The US RST company mainly uses metal tantalum wire composite fabrics to achieve lightweight and highefficiency protection against $\mathrm{X}$ and gamma rays, but it faces problems such as the high cost of metal tantalum and complex tantalum wire processing technology. The American INFAN company and the German MI company add nano-heavy metal alloy powder to the fiber to prepare functional clothing for radiation protection to achieve the same protective effect. The weight is only about $30 \%$ of the traditional lead clothing, but the alloy still contains a certain proportion of metallic lead. Nanoalloy powders are complex to process and difficult to disperse in polymers, and fiber-forming and processing are difficult. The Japanese company adopts the skin-core structure melt spunbond spinning technology, taking into account the high addition of powder and the mechanical properties of non-woven materials, mainly by increasing the thickness of the fabric to improve the protective effect.

Domestic China Institute of Radiation Protection, Hubei Huaqiang Technology Co., Ltd., Beijing University of Chemical Technology, and other institutions have researched lead-free radiation protection materials[1,2,3]. The research focus of leadfree radiation protection materials is mainly on glass, Rubber, board, and other materials are added with heavy metal powder, which is mainly used for the protection and isolation of the radiation source body. And there is less development of individual radiation protection fibers and clothing fabrics. There is a lack of in-depth research on lightweight textile materials for lead-free radiation protection. Therefore, it is particularly necessary to conduct systematic research on the selection and modification of lead-free radiation protection materials, fiber molding technology, textile material structure design, and evaluation methods.

This article lacks in-depth research on lightweight textile materials for lead-free radiation protection. It is particularly necessary to conduct systematic research on the selection and modification of lead-free radiation protection materials, fiber molding technology, textile material structure design, and evaluation methods.

\footnotetext{
${ }^{\mathrm{a}}$ Corresponding author: lihonghui@cirp.org.cn
} 


\section{Principle and Method}

Protective clothing has various structural forms. As shown in Figure 1, a representative type of protective clothing is mainly composed of an inner layer, an outer structural layer, and a protective layer with multiple protective functions. The shielding material of the protective layer has various structures. When X-rays with low energy represented by $150 \mathrm{kV}$ interact with matter, the main use of high atomic number materials is to reduce the amount of energy of X-rays through the photoelectric effect of X-rays and Compton scattering.

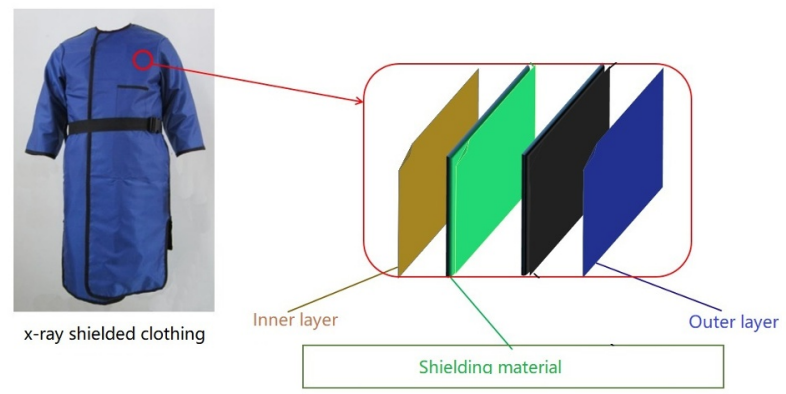

Figure 1 Schematic diagram of X-ray shielding protective clothing and its material structure

For personnel X-ray protection, it can be expressed as a reduction coefficient relationship to the dose, which is expressed as follows:

$$
\mathrm{K}=\frac{\mathrm{Dx_{0 }}}{\mathrm{Dx}_{\mathrm{i}}}
$$

Where $\mathrm{K}$ is the protection weakening coefficient of the protective material against X-rays; Dxo represents the dose rate without X-ray protective materials $(\mathrm{mSv} / \mathrm{h})$; Dxi represents the dose rate with $\mathrm{X}$-ray protective materials $(\mathrm{mSv} / \mathrm{h})$. The protective performance of the material can be determined by determining the dose rate relationship before and after the protection $[4,5]$.

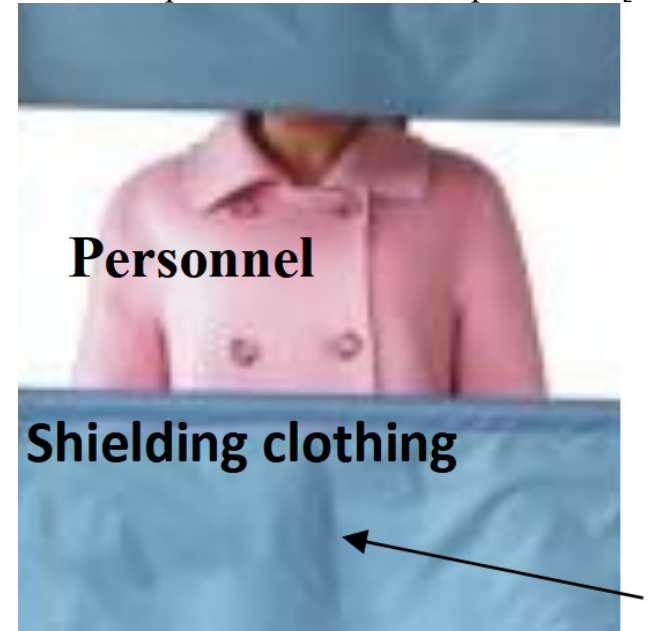

Xay

Figure 2 Schematic diagram of the shielding calculation model

The protective layer usually can use a single metal textile or additive materials, and can also use combined or mixed materials. To reduce the radiation hazards of material development, the Monte Carlo method is studied to simulate X-rays, the researched protective shielding materials, and the three-body physical model of the personnel, and the reasonable material plan is evaluated with formulas.

To reduce unnecessary analysis, studied and trialproduced the feasibility of metallic bismuth and tungsten materials as textile materials. Preliminary tests have shown that three structures of single metal (as shown in Figure 3), layered metal, and metal mixture can be realized. Therefore, the simulated protective shielding materials are used to carry out protective performance research based on these three determined ranges.

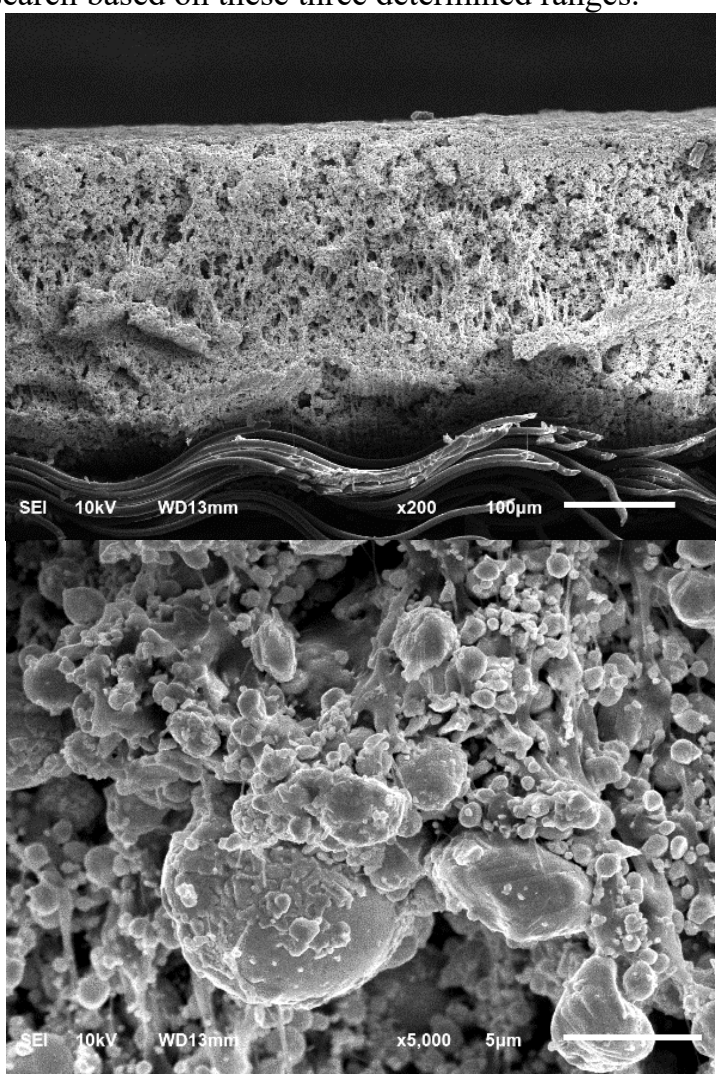

Figure 3 SEM image of coated fabric with added metal powder

\section{Calculation and Analysis results}

\subsection{Single metal layer result}

Considering that the application range of $150 \mathrm{kV}$ X-ray is different from the filter device, the X-ray energy spectrum is different. Generally speaking, there are many kinds of X-ray equipment whose main energy spectrum is distributed at $150 \mathrm{keV}$ and below. The 150 $\mathrm{kV} \mathrm{X}$-ray is investigated according to the target substance. There are obvious differences in the different energy spectra of the filter materials, but some investigations show that $80 \%$ or more of the energy spectra are mostly concentrated in $60 \sim 120 \mathrm{keV}$. The simulation carried out $150 \mathrm{keV}$ and the following is divided into $60 \mathrm{keV}, 70 \mathrm{keV}, 80 \mathrm{keV}, 90 \mathrm{keV}, 100 \mathrm{keV}$, $110 \mathrm{keV}, 120 \mathrm{keV}, 150 \mathrm{keV}$. The dose rate of X-ray shielded by a textile protective material. Figure 4 shows the material model shown in Figure 3, the shielding 
material is $2 \mathrm{~kg} / \mathrm{m}^{2}$ single metal tungsten, and the X-ray dose rate under $150 \mathrm{keV}$ is used for shielding. Figure 5 shows the X-ray dose rate under $150 \mathrm{keV}$ is shielded with a $2 \mathrm{~kg} / \mathrm{m}^{2}$ single metal bismuth material.
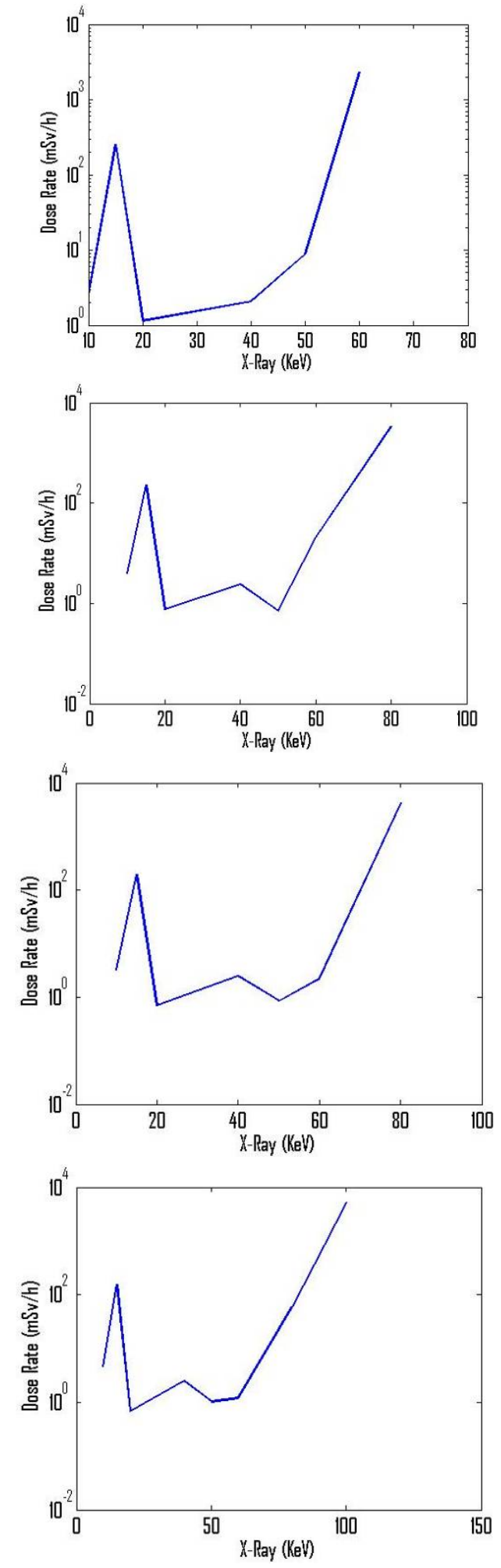
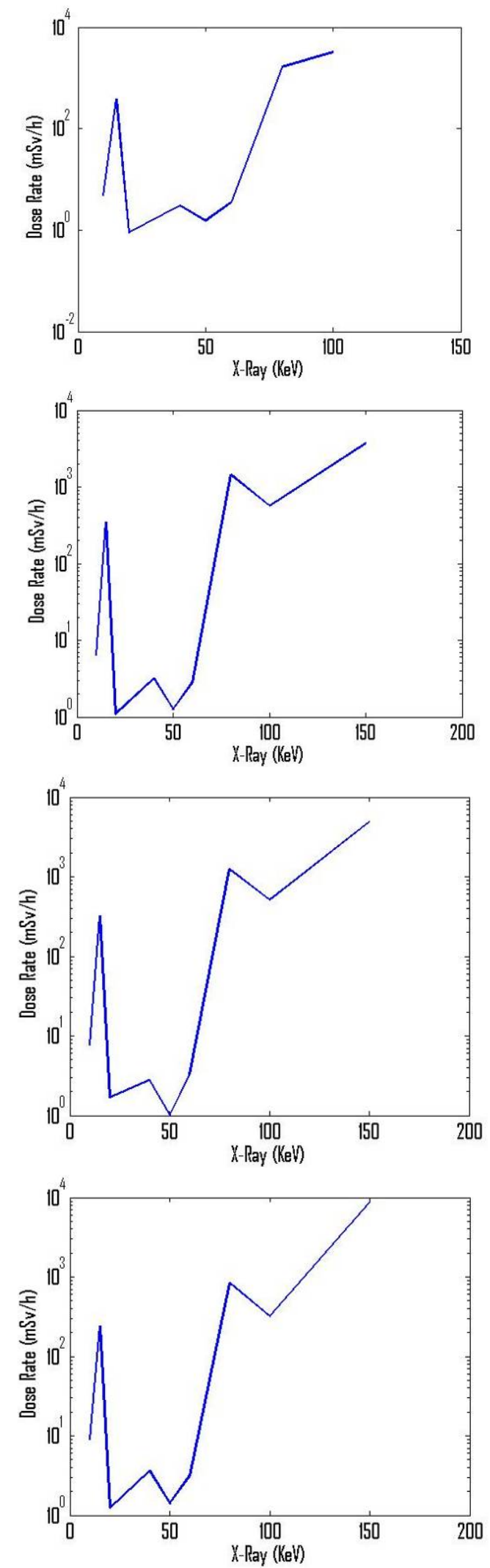

Figure 4 Use $2 \mathrm{~kg} / \mathrm{m}^{2}$ tungsten material as shielding X-ray dose rate under $150 \mathrm{keV}$ 

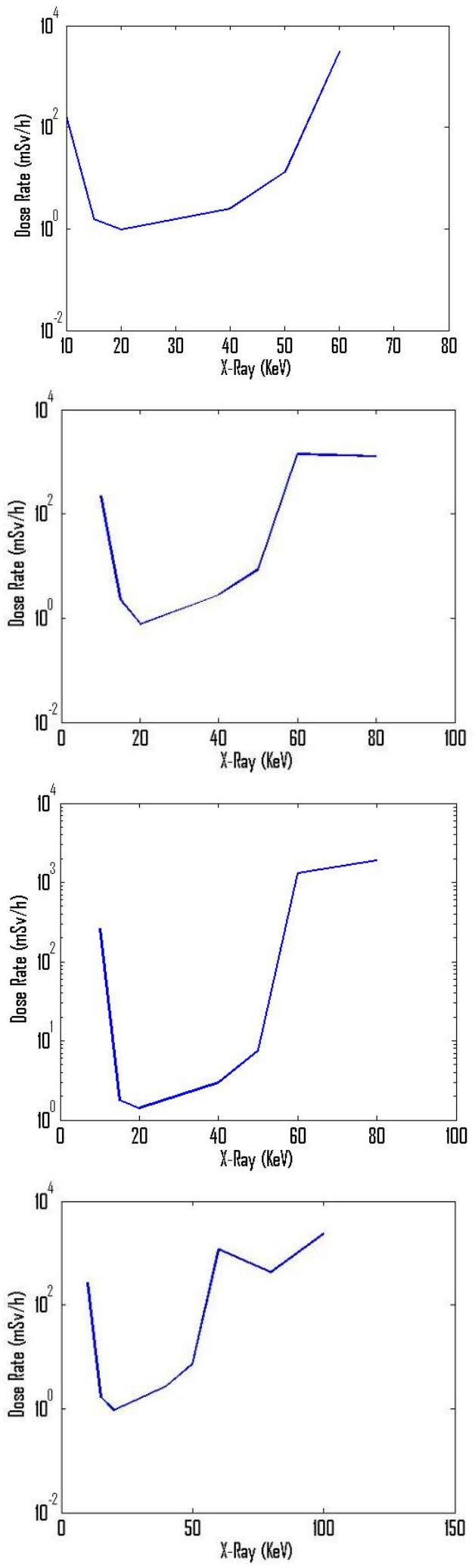
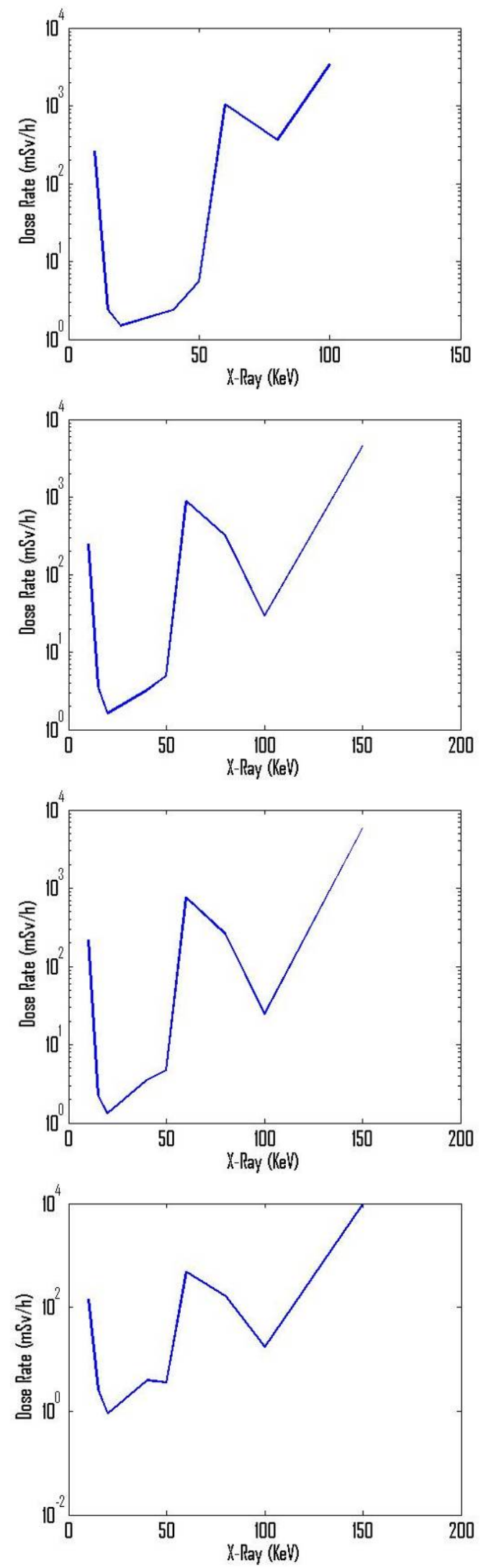

Figure 5 Shield the dose rate of X-rays under $150 \mathrm{keV}$ with 2 $\mathrm{kg} / \mathrm{m}^{2}$ bismuth material

According to Figures 4 and 5, the protective performance of concern is obtained, as shown in Figure 6 . If the X-ray energy component is considered at 150 $\mathrm{keV}$, the protective weakening coefficient of all heavy metal materials against X-ray degradation can only reach 
between 1.22 and 1.26. The attenuation coefficient of 1.5 cannot be achieved, and the protection performance of $\mathrm{X}$-ray reduction is not obvious. For the energy spectrum distribution, the main weight is concentrated below 100 $\mathrm{keV}$, and the X-ray dose rate can be reduced by 1.5 by relying on $\mathrm{Wu}$ metal. If it is bismuth metal, it is possible to ensure that the X-ray energy is mainly concentrated at $70 \mathrm{keV}$ and below to achieve the attenuation of X-rays to 1.5 .

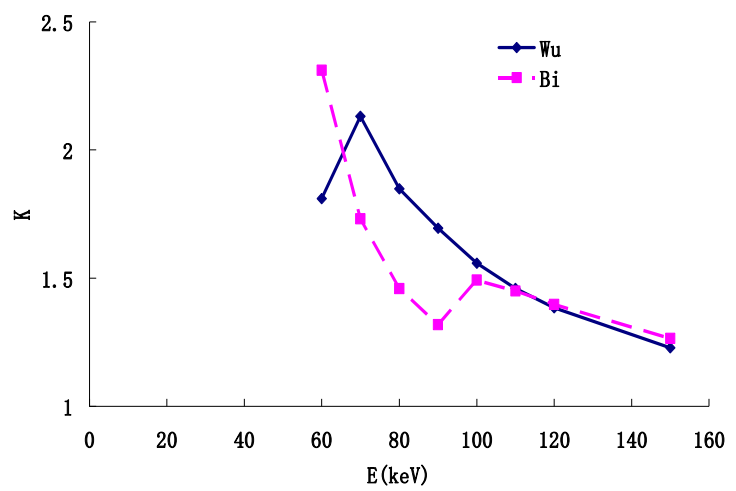

Figure 6 The ability to protect single-energy X-rays below 150 $\mathrm{keV}$ with $2 \mathrm{~kg} / \mathrm{m}^{2}$ tungsten or bismuth textile materials

\subsection{Double-layer metal results}

The protective fabric shielding material can also be an interlayer of two metal materials arranged in sequence. For this reason, the same model as shown in Figure 2 is adopted, and the shielding material adopts a double metal layer structure. The material uses a mass ratio of 1: 1 to simulate $\mathrm{Bi} / \mathrm{Wu}$ double-layer shielding and $\mathrm{Wu} / \mathrm{Bi}$ double-layer shielding. $150 \mathrm{keV}$ and below, covering 60 $\mathrm{keV}, 70 \mathrm{keV}, 80 \mathrm{keV}, 90 \mathrm{keV}, 100 \mathrm{keV}, 110 \mathrm{keV}, 120$ $\mathrm{keV}, 150 \mathrm{keV}$ The dose rate of X-ray shielded by textile protective materials, and then the same as a single layer of metal, the results are sorted out. The X-ray dose rate of the two layers of metal bismuth and tungsten arranged in different order is reduced. Figure 7 shows that the shielding material is $2 \mathrm{~kg} / \mathrm{m}^{2}$ metal tungsten and bismuth double-layer material for shielding. The X-ray dose is reduced to under $150 \mathrm{keV}$.

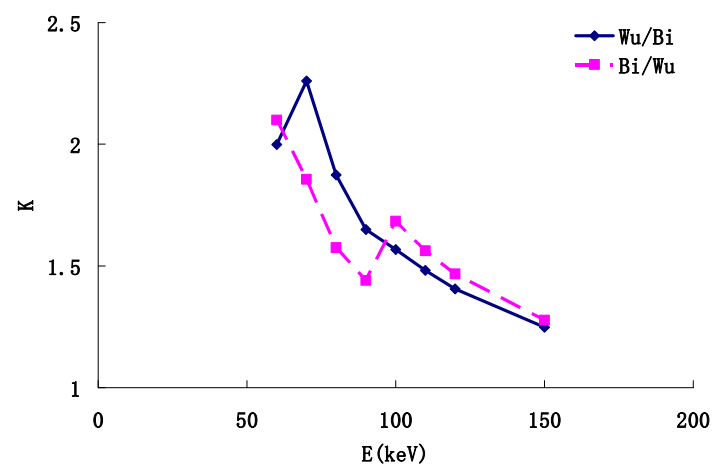

Figure 7 The attenuation coefficient of single-energy X-rays under different orders of $2 \mathrm{~kg} / \mathrm{m}^{2}$ tungsten-bismuth two-layer metal material

As shown in Figure 7, if the X-ray energy component is considered at $150 \mathrm{keV}$, it is also impossible for all heavy metal materials to achieve the ability to weaken X-rays by 1.5 . If the energy of X-rays is mainly concentrated below $100 \mathrm{keV}$, it is possible to achieve the requirements that the dose rate of X-ray reduce by 1.5. Meanwhile, because of the difference in scattering and resonance absorption of different materials, even the same material, different combinations are different. Besides, considering that in clothing textile applications, separate metal or layered metal and its different inter-layer weaving are not ideal fabrics, so consider the mixed composite situation. To this end, the feasibility of metal mixing research has been carried out, and a simulation study has been done to calculate and analyze the difference between it and the layering, which can lay the foundation for the subsequent realization of the ideal fabric.

\subsection{Metal hybrid structure results}

The model is the same as in Figure 2. The shielding material adopts a bimetallic mixed structure. The mixed materials are determined by mass ratios, and the $\mathrm{Wu}$ : $\mathrm{Bi}$ ratios are respectively $0.2: 0.8,0.5: 0.5,0.8: 0.2$ metal mixing. Simulate radiation $150 \mathrm{keV}$ and below, covering $60 \sim 120 \mathrm{keV}$ and $150 \mathrm{keV}$ shielding performance dose rate calculation. Figure 7 shows the model of Figure 3, the shielding material is $2 \mathrm{~kg} / \mathrm{m}^{2}$, and the metal tungstenbismuth mixing ratio is $0.2: 0.8,0.5: 0.5,0.8: 0.2$. Three metal mixed structures (recorded as WuBiMix1, WuBiMix2, WuBiMix3 in the figure). The X-ray attenuation coefficient under the mixed material shielding $150 \mathrm{keV}$.

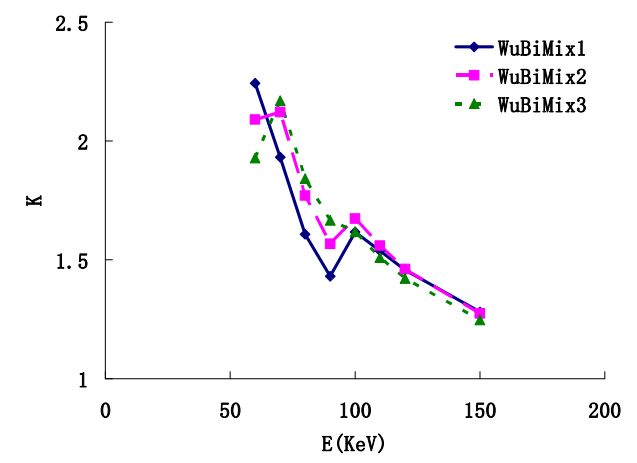

Figure 8 The areal density of $2 \mathrm{~kg} / \mathrm{m}^{2}$, the mixed material of $\mathrm{Wu}$ and $\mathrm{Bi}$ mass ratio 0.8: 0.2 shields the $\mathrm{X}$-ray attenuation ability under $150 \mathrm{keV}$

According to the results of Fig. 8, if the X-ray energy component is considered at $150 \mathrm{keV}$, it is also impossible for mixed metal materials to achieve the possibility of a weakening factor of 1.5 for X-rays.

\section{Analysis and Discussion}

The performance of protective clothing of different materials is normalized to the relationship of $\mathrm{Bi}$ performance, and Figure 8 is obtained. The results show that the higher the proportion of bismuth when the radiation component is low in energy, the better the protective performance. As the energy increases beyond $70 \mathrm{keV}$, the more tungsten materials, the better the protective performance. Compared with a single metal, 
tungsten has poorer protection performance below 70 $\mathrm{keV}$. Between $70 \sim 120 \mathrm{keV}$, the protection performance of tungsten is much better than that of $\mathrm{Bi}$. When the $\mathrm{X}$ ray energy is higher than $120 \mathrm{keV}$, the protection performance of bismuth begins to be better than that of metal tungsten. Except for the X-ray energy lower than $70 \mathrm{keV}$ in the double-layer metal, interlayer material has better protection performance than a single metal bismuth material. When the energy is lower than $70 \mathrm{keV}$ $\mathrm{X}$-ray, the metal bismuth serves as the first layer of protection for the protection below $70 \mathrm{keV}$. The performance is better than tungsten metal, and the metal bismuth is used as the first layer when low energy is dominant. When X-rays are above $70 \mathrm{keV}$, tungsten as the first layer material has obvious advantages in protection performance, and the overall protection performance is better than single metal bismuth and bismuth as the first layer protection material. The performance of the mixed material is similar to that of the double-layer metal material, except that it is less than $70 \mathrm{keV}$, its protection performance is also better than that of a single metal bismuth material. If the ray energy is between $70 \sim 90 \mathrm{keV}$, the mass ratio of tungsten to bismuth is $0.8: 0.2$. When the energy is high, the protection performance is best when the mass ratio of tungsten to bismuth is 0.5 : 0.5 , and when the X-ray energy is less than $70 \mathrm{keV}$, the mass ratio of tungsten to bismuth is 0.2 : 0.8 as superior.

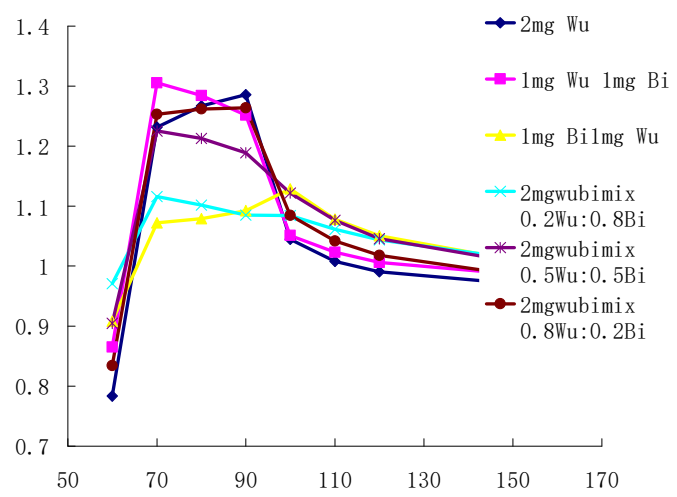

Figure 9 Comparison of protection effect under different energy

\section{Conclusion}

In this paper, combined with the feasibility of textile materials, the Monte Carlo method is used to analyze the $150 \mathrm{kV}$ typical X-ray protection capacity of single-layer metal, double-layer metal, and different proportions of metal mixed textile materials. The results show that if the X-ray composition is mainly below $70 \mathrm{keV}$, then Regardless of the method used, metallic bismuth should account for the main proportion or directly use metallic bismuth. Above $70 \mathrm{keV}$, the protective performance of a single metal bismuth material is weaker than that of double-layer or mixed metal materials. For this reason, mixed materials should be used. Among them, the ratio of tungsten and bismuth is 0.5 : 0.5 or $0.8: 0.2$. For good protection performance and comprehensive low-energy protection effect, it is a better choice to use tungsten and bismuth ratio of 0.5 : 0.5 .

\section{Acknowledgement}

This research was supported by Foundation of The National Key Research and Developmeng program of China (2018YFC0810301).

\section{References}

1. S. Fu, Application of MCNP to Study the shielding Effect of Zinc Bromide Solution,[R], Progress in NUCLEAR and TECHNOLOGY, Vol. 1, p.98101(2011)

2. S. Fu, E. Yao, Weibing Yang, etc. Study on shielding material (sands) for disused high activity radioactive sources conditioning $[\mathrm{J}]$. Radiation protection. 2018(06).

3. S. Fu, etc. Shielding Calculation on Spent High Activity Radioactive Sources Conditioning [J]. Radiation protection newsletter. 2008(05).

4. General Administration of Quality Supervision, Inspection and Quarantine of the People's Republic of China, Basic Standards for Ionizing Radiation Protection and Radiation Source Safety [S], GB18871-2002.

5. S. Fu etc, A structurally optimized movable liquid outflow monitoring device[P]. ZL201420692457.9. 